\title{
A STEREOLOGICAL ESTIMATION OF GONAD VOLUME AND OOCYTE NUMBER IN A SPECIMEN OF A NATIVE CLAM (VENERUPIS PULLASTRA)
}

\author{
Pablo Alvarez Fariñ ${ }^{1}$ And Ximo Gual-ArnaU ${ }^{2}$ \\ ${ }^{1}$ Dpto. de Fisiloxía. Facultade de Farmacia, Universidade de Santiago de Compostela, 15782-Santiago de \\ Compostela, Spain, ${ }^{2}$ Department of Mathematics, Universitat Jaume I, 12071-Castelló, Spain \\ e-mail: fspablo@usc.es,gual@mat.uji.es \\ (Accepted June 15, 2006)
}

\begin{abstract}
We obtained the gonad volume and oocyte number in a single Venerupis pullastra (Montagu, 1803) using unbiased stereological estimators. Since the gonad is not a fixed anatomical structure and is merged with other organs in the clam, we used new variance expressions to study the efficiency of these estimators, where the isotropy condition is not required for the covariogram.
\end{abstract}

Keywords: fecundity, gonad volume, oocyte number, stereology, unbiased estimators, variance, Venerupis pullastra.

\section{INTRODUCTION}

Unbiased Stereological estimators require that the probes or test systems (section planes, points, etc.) are positioned at random in the reference space; moreover, for a number of practical reasons as well as for its superior efficiency, systematic sampling has been performed in design-based stereology: specimens are sectioned by sets of parallel planes, fields of vision on a section are selected roughly equidistant and on each field of vision a test system with regularly spaced points is placed (a recent reference is Baddeley and Jensen, 2005).

Most of the studies considered in the literature to describe the reproduction cycle of different types of clams obtain the periods of egg-laying and the determining factors in the reproductive strategies of the clams (these factors are, mainly, temperature and food disposition, e.g., Beukema et al. 2001).

This paper deals with the estimation of the volume of the gonad in a clam (Venerupis pullastra) and with the estimation of the total number of oocytes within the gonad. This number is taken to be a measure of the fecundity of the clams. The total volume is obtained with a standard Cavalieri estimator based on point counting, as described in the second section. The third section discusses the estimation of the variance due to the point counting using a geometric covariogram, and the fourth section considers the variance of the Cavalieri estimator for total volume. Finally, the fifth section deals with the estimation of the total number of oocytes as a product of gonad volume and number density.
Since gonad volume and oocyte number are relevant quantities in order to analyse the gonad development stage and egg production rate, our purpose is to estimate them in a bivalve with a diffused and changing organ (the gonad) and to present recent and new variance expressions to study the efficiency of these estimators.

\section{MATERIALS}

Histology. An individual specimen of the clam Venerupis pullastra (shell length $18.8 \mathrm{~mm}$; weight $1.08 \mathrm{~g})$ was utilised. The soft tissues were removed and fixed in Bouin's fluid. Then, the tissues were dehydrated in an alcohol series and embedded in plastic in order to prevent loss of tissue. Histological sections $(5 \mu \mathrm{m})$ were stained in Wheatley's trichrome.

To estimate volume, images were obtained at $1 \times$ magnification. Each of these images shows a whole section of the clam: visceral mass, gonad, digestive gland and other soft tissues (foot, gills and syphons). To estimate oocyte number, images were obtained at $20 \times$ magnification in the microscope.

\section{GONAD VOLUME ESTIMATION FROM CAVALIERI SECTIONS}

To estimate the gonad volume $V$ of our Venerupis pullastra, we have fixed a convenient sampling axis $O x$ and then

$$
V=\int_{-\infty}^{\infty} A(x) d x
$$


where $A(x)$ denotes the area of the intersection between the gonad of the Venerupis pullastra and a plane normal to $O x$ at a point on the abscissa $x$. We estimate $V$ from systematic plane sections a distance $T=0.305 \mathrm{~mm}$ apart, normal to the sampling axis at the abscissas $\{z+k T, k \in \mathbb{Z}\}$, where $z=U T$ and $U$ is a uniform random number in the interval $(0,1)$. The Cavalieri estimator of $V$, which is easily shown to be unbiased, is

$$
\tilde{V}=T \sum_{k \in \mathbb{Z}} A(z+k T)=T\left(A_{1}+A_{2}+\cdots+A_{n}\right),
$$

where $A_{1}, \ldots, A_{n}$ are the section areas of the gonad of the Venerupis pullastra in sequential order (in our case $n=11)$.

Since the gonad is merged with the rest of the organs in the Venerupis pullastra body it is not easy to obtain the section areas $A_{1}, \ldots, A_{n}$, so we have estimated them using a point-counting estimator.

To estimate the area $A$ of the planar domain $D$ we use systematic sampling at the vertices of a rectangular grid of sides $T_{1}=u=\sqrt{2.3 / 4}=0.7583 \mathrm{~mm}$ and $T_{2}=u=\sqrt{2.3 / 4}=0.7583 \mathrm{~mm}$; then

$$
\hat{A}=u^{2} \sum_{i=-\infty}^{\infty} \sum_{j=-\infty}^{\infty} 1_{D}\left(z_{1}+i u, z_{2}+j u\right)=u^{2} P,
$$

where $z_{1}$ and $z_{2}$ are independent uniform random variables in the interval $[0, u), 1_{D}$ is the indicator function of $D$ and $P$ denotes the number of test points (namely grid vertices) hitting $D$.

Now, the estimator used for $V$ is

$$
\hat{V}=T \sum_{k=1}^{n} \hat{A}(z+k T)=T u^{2}\left(P_{1}+P_{2}+\cdots+P_{n}\right),
$$

where $P_{i}$ denotes the number of points hitting the i-th gonad section.

The results obtained in our estimation were

$$
\begin{gathered}
\hat{V}=T \cdot u^{2}(20+28+28+23+24+35+22+11+ \\
8+5+3)=207 T u^{2} \mathrm{~mm}^{3}=36.302625 \mathrm{~mm}^{3} .
\end{gathered}
$$

To predict the efficiency of this estimation we will consider the variance of the estimators given in Eqs. 2 and 3. That is, under some elementary mathematical assumptions we have

$$
\operatorname{Var}(\hat{V})=\operatorname{Var}(\tilde{V})+T \sum_{i=1}^{n} \sigma_{i}^{2},
$$

where $\operatorname{Var}\left(\hat{A}_{i}\right)=\sigma_{i}^{2}$. The last term on the right side of the preceding equation is the contribution of the nugget variance to the variance.
The coefficient of error, $C E()=\sqrt{\operatorname{Var}()} / E()$, where $E$ is expected value, reveals the accuracy of the estimator; therefore, we are interested in the prediction of $C E$ in our case. To obtain this prediction we will first consider the nugget variance due to the point-counting estimator of planar area and later on the variance of the Cavalieri estimator for volume.

\section{VARIANCE OF THE POINT-COUNTING ESTIMATOR FOR PLANAR AREA}

To approximate the variance of the estimator given in Eq. 3, we will consider two variance representations based on the geometric covariogram of $D$. The geometric covariogram of $D$ is a function $g: \mathbb{R}^{2} \longrightarrow \mathbb{R}$ defined as

$$
\begin{aligned}
g(x, y) & =\int_{\mathbb{R}} \int_{\mathbb{R}} f\left(z_{1}, z_{2}\right) f\left(z_{1}+x, z_{2}+y\right) d z_{1} d z_{2} \\
& =\text { Area }(D \cap(D+(x, y)))
\end{aligned}
$$

where $f\left(z_{1}, z_{2}\right)=1_{D}\left(z_{1}, z_{2}\right)$ and $D+(x, y)$ is the translation of $D$ by the vector $(x, y)$. That is, $g(x, y)$ is the area of the intersection between $D$ and a copy of $D$ translated by $(x, y)$.

In general, and especially in our case where the gonad is an irregular domain, the geometric covariogram depends on the vector $(x, y)$ and is anisotropic. Therefore, we will consider the approach of Gual-Arnau and Cruz-Orive, 2006 and use as the variance approximation the expression

$$
\operatorname{Var}(\hat{A})=\frac{-u^{3}}{6} \sum_{k \in \mathbb{Z}}\left(\frac{\partial g}{\partial y}(k u, 0)+\frac{\partial g}{\partial x}(0, k u)\right) .
$$

Now, we approximate the partial derivatives by

$$
\begin{aligned}
& \frac{\partial g}{\partial y}(k u, 0) \approx \frac{g(k u, u)-g(k u, 0)}{u}, \\
& \frac{\partial g}{\partial x}(0, k u) \approx \frac{g(u, k u)-g(0, k u)}{u} .
\end{aligned}
$$

On the other hand, the unbiased estimators of the required covariogram values $g\left(k_{1} u, k_{2} u\right)$ are

$$
u^{2} C_{k_{1}, k_{2}}=u^{2} \sum_{i=-\infty}^{\infty} \sum_{j=-\infty}^{\infty} f_{i j} f_{i+k_{1}, j+k_{2}}
$$

where $f_{i j}=1_{D}\left(z_{1}+i u, z_{2}+j u\right)$. 
Then,

$$
\operatorname{Var}_{1}(\hat{A}) \approx \frac{-u^{4}}{6} \sum_{k \in \mathbb{Z}}\left(\left(C_{k, 1}-C_{k, 0}\right)+\left(C_{1, k}-C_{0, k}\right)\right)
$$

On the other hand, if we assume that $g$ is isotropic, a known approximation variance states (see CruzOrive, 1993)

$$
\begin{aligned}
& \operatorname{Var}_{2}(\hat{A}) \\
\approx & 0.0569 u^{4}\left(6 C_{0,0}-4\left(C_{1,0}+C_{0,1}\right)-\left(C_{2,0}+C_{0,2}\right)\right) .
\end{aligned}
$$

Now we approximate the nugget variance defined in Eq. 6 using Eq. 12 ( $g$ is anisotropic) and Eq. 13 ( $g$ is assumed to be isotropic).

$$
\begin{aligned}
T \sum_{i=1}^{n} \sigma_{i}^{2} \approx & T \sum_{i=1}^{n} \operatorname{Var}_{1}\left(\hat{A}_{i}\right) \\
= & T u^{4}(6.83+5.67+7.33+4+5+7 \quad(14) \\
& +6.33+1.83+3.5+1.17+0.83) \\
= & 49.49 T u^{4} . \\
T \sum_{i=1}^{n} \sigma_{i}^{2} \approx & T \sum_{i=1}^{n} \operatorname{Var}_{2}\left(\hat{A}_{i}\right) \\
= & T u^{4}(2.67+3.02+4.15+4.6+3.87 \\
& +3.02+2.28+1.82+1.88+1.08+0.57) \\
= & 28.96 T u^{4} .
\end{aligned}
$$

Note that Eq. 14 and Eq. 15 are based on the actual measurement of the specimen.

From Eqs. 2, 14 and 15, we obtain approximations of the coefficient of error in the estimation of planar area by point-counting; for instance, the coefficient of error of $\hat{A}_{1}$ is, using Eq. 14 and Eq. 15 , respectively, $C E_{1}\left(\hat{A}_{1}\right) \approx \sqrt{6.83} / 20=0.13$ and $C E_{2}\left(\hat{A}_{1}\right) \approx \sqrt{2.67} / 20=0.082$.

In Fig. 1 we have the histological section which corresponds to the area $A_{5}$. In Fig. 2 the points of the grid which lie in the gonad appear in green $P_{5}=24$. In Fig. 3 we have the points which appear in green in Fig. 2. The number of black points is $C_{1,1}$, that is, the black points are the intersection between the grid of green points and a copy of this grid translated by the vector $(1,1)$.

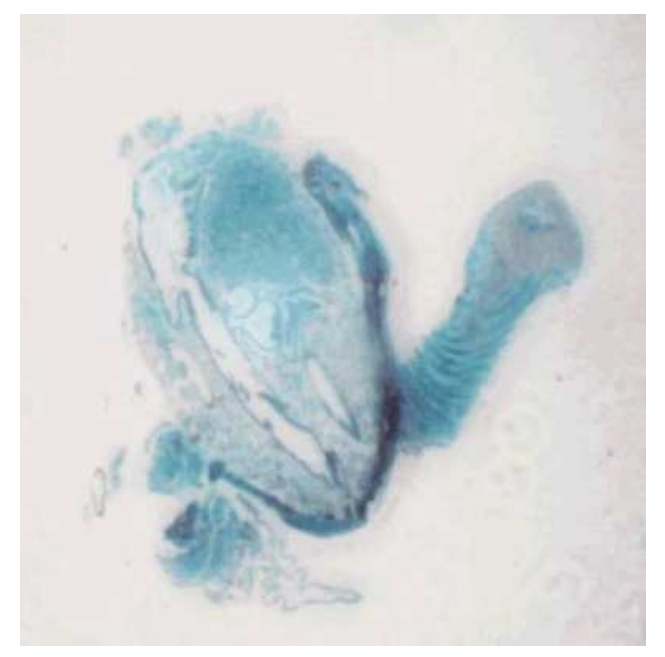

Fig. 1. An example of an image obtained from a histological section of the clam for volume estimation.

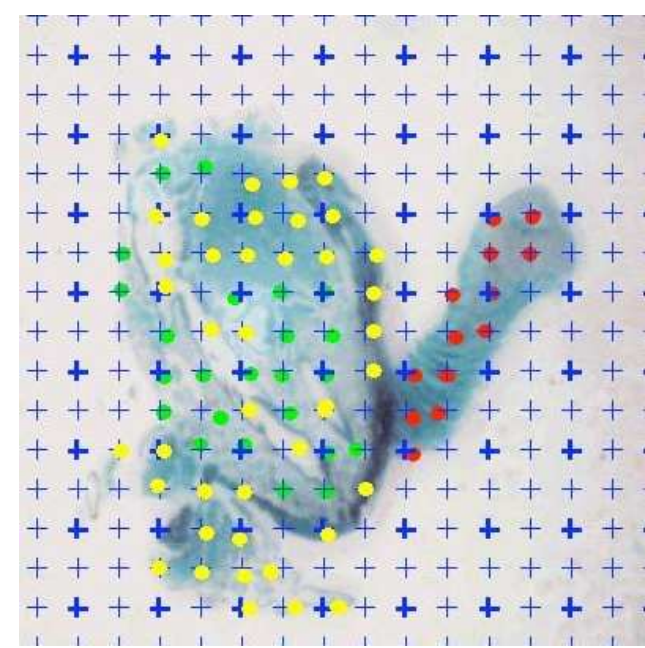

Fig. 2. The points of the grid which lie in the gonad appear in green and they give an estimate of $A_{5}$. Red dots correspond to clam's foot and yellow dots to visceral mass.

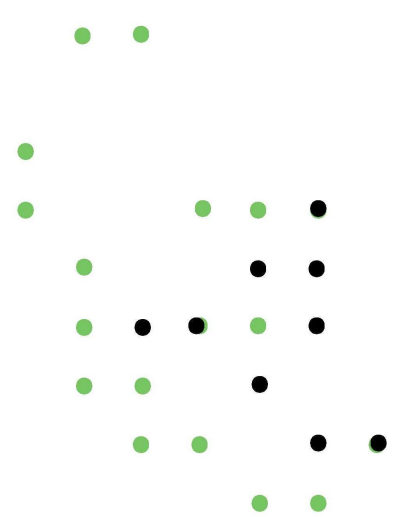

Fig. 3. The number of black points is $C_{1,1}=9$; that is, the black points are the intersection between the grid of green points and a copy of this grid translated by the vector $(1,1)$. 


\section{VARIANCE OF THE CAVALIERI ESTIMATOR FOR VOLUME}

Here we approximate the variance of the volume estimator using a formula based on the covariogram of the measurement function $A(x)$

$$
g(y)=\int_{\mathbb{R}} A(y) A(y+x) d y .
$$

This formula is obtained in Gual-Arnau and CruzOrive, 2006 and, since the gonad is an irregular domain in the specimen, we will use the expression

$$
\begin{aligned}
& \operatorname{Var}(\tilde{V}) \approx \\
& \approx-\frac{T^{2}}{3}\left(g^{\prime}(0)+\frac{T}{4}\left(g^{\prime \prime}(0)+2 \sum_{k=1}^{\infty} g^{\prime \prime}(k T)\right)\right)
\end{aligned}
$$

Now, the derivatives of the covariogram are approximated by

$$
\begin{aligned}
& g^{\prime}(0) \approx \frac{-g(2 T)+4 g(T)-3 g(0)}{2 T}, \\
& g^{\prime \prime}(0) \approx \frac{g(2 T)-2 g(T)+g(0)}{T^{2}}, \\
& g^{\prime \prime}(j T) \frac{g((j+1) T)-2 g(j T)+g((j-1) T)}{T^{2}}, \\
& j=1, \ldots, n-1 .
\end{aligned}
$$

Then,

$$
\begin{gathered}
\operatorname{Var}(\tilde{V}) \approx \frac{T}{12}(3 g(0)-4 g(T)+g(2 T)) \\
+\frac{T}{6} g((n-1) T)
\end{gathered}
$$

The first term on the right side of the preceding equation is a classical approximation formula for variance (see Gundersen and Jensen, 1987).

Now, using the estimators of the required covariogram values $g(k T)$,

$$
T C_{k}=T \sum_{i=1}^{n-k} \hat{A}_{i} \hat{A}_{i+k},
$$

and considering local errors (see Cruz-Orive, 1999), we obtain

$$
\begin{aligned}
\operatorname{Var}(\hat{V}) \approx & \frac{T^{2}}{12}\left(3\left(C_{0}-\sum_{i=1}^{n} \sigma_{i}^{2}\right)-4 C_{1}+C_{2}\right) \\
& +T^{2} \sum_{i=1}^{n} \sigma_{i}^{2}+\frac{T^{2}}{6} C_{n-1} .
\end{aligned}
$$

In our case (see Eq. 5) $C_{n-1}=C_{10}=20 * 3 u^{4}=$ $60 u^{4}, C_{0}=5001 u^{4}, C_{1}=4535 u^{4}$ and $C_{2}=3849 u^{4}$.

Now, from Eq. 23 but using Eq. 14 and Eq. 15 we have two variance approximations

$$
\begin{aligned}
\operatorname{Var}_{1}(\hat{V}) & \approx T^{2} u^{4}\left(\frac{563.53}{12}+49.49+10\right) \\
& =106.45 T^{2} u^{4}
\end{aligned}
$$

and

$$
\begin{aligned}
\operatorname{Var}_{2}(\hat{V}) & \approx T^{2} u^{4}(52.1+28.96+10) \\
& =91.05 T^{2} u^{4}
\end{aligned}
$$

Finally, from the preceding approximations and from Eq. 5 we have

$$
\begin{aligned}
C E_{1}(\hat{V}) & \approx \frac{\sqrt{106.45} T u^{2}}{207 T u^{2}} \\
& =0.05
\end{aligned}
$$

and

$$
\begin{aligned}
C E_{2}(\hat{V}) & \approx \frac{\sqrt{91.05} T u^{2}}{207 T u^{2}} \\
& =0.046 .
\end{aligned}
$$

\section{ESTIMATION OF OOCYTE NUMBER BY THE CAVALIERI-DISECTOR DESIGN}

The total number of oocytes within the gonad will be estimated indirectly via the corresponding number density $N=V N_{V}$, where $V$ represents the volume of the gonad (estimated in the preceding sections) and $N_{V}$ the oocyte number per unit volume.

We estimate the oocyte number on specially treated adjacent series by systematically subsampled disectors; the procedure was carried out on a monitor screen with the aid of the CAST Grid system (Olympus Denmark). The estimator that we use is

$$
\hat{N}_{V}=\frac{\sum Q^{-}}{a^{\prime} h \sum P^{\prime}} \mathrm{mm}^{-3},
$$

where $h$ represents disector height, which in our case is the same for all slices $h=0.005 \mathrm{~mm}, P^{\prime}$ the number of upper right corners of the sampling frames hitting the relevant reference space in the upper disector face within the same slice, $a^{\prime}$ the area of the counting frame $\left(a^{\prime}=0.144 \mathrm{~mm}^{2}\right)$ and $\sum Q^{-}$the total number of oocytes counted in all the disectors. 
Then, from Eq. 5 and Eq. 28 we obtain

$$
\begin{aligned}
\hat{N}= & \frac{T a \sum_{i=1}^{11} P_{i} \sum Q^{-}}{a^{\prime} h \sum P^{\prime}} \\
= & \frac{36.3026205 * \sum Q^{-}}{0.144 * 0.005 * 68} \\
= & 741.475(25+39+47+39+39+44+ \\
& +28+18+10+13+5) \\
= & 741.475 * 307=227633 .
\end{aligned}
$$

The quantity that $\sum Q^{-}$is multiplied by in Eq. 29 is not a constant; however, this equation is basically equivalent to an alternative expression arising from the fractionator idea. From this expression, which is detailed in Cruz-Orive et al. (2004, p. 27), we obtain

$$
C E(\hat{N})=C E\left(\sum Q^{-}\right)
$$

at least approximately.

Now, the square of the coefficient of error of the estimator $\hat{N}$ may be approximated by (Cruz-Orive and Geiser, 2004 and Cruz-Orive, 1999)

$$
\begin{aligned}
& C E^{2}(\hat{N})= \\
= & \alpha(h / T, q) \frac{3\left(C_{0}-\sum_{i=1}^{n} \mu_{i}^{2}\right)-4 C_{1}+C_{2}}{\left(\sum Q^{-}\right)^{2}}+\frac{\sum_{i=1}^{n} \mu_{i}^{2}}{\left(\sum Q^{-}\right)^{2}},
\end{aligned}
$$

where $\alpha(h / T, q)$ is a constant depending on the sampling fraction $h / T$ and the smoothness constant of the measurement function $q, C_{k}=\sum_{j=1}^{n-k} f_{i} f_{i+k}\left(f_{i}\right.$ represents the estimation number of particles in the ith primary slice) and $\mu_{i}^{2}$ is the conditional variance of $f_{i}$ given the true number of particles.

To simplify the estimate of $\sum_{i=1}^{n} \mu_{i}^{2}$ we may resort to the homogeneous Poisson particle model (CruzOrive and Geiser, 2004), and we have

$$
\begin{aligned}
& C E^{2}(\hat{N})= \\
& =\alpha(h / T, q) \frac{3\left(C_{0}-\sum Q^{-}\right)-4 C_{1}+C_{2}}{\left(\sum Q^{-}\right)^{2}}+\frac{1}{\sum Q^{-}} .
\end{aligned}
$$

Note that only the local error contribution uses a special assumption for the arrangement of the oocytes (approximately Poisson).

Now an estimate of $q$ is given by the Kieu-Souchet formula (Kieu et al., 1999),

$$
\hat{q}=\frac{1}{2 \log 2} \log \left(\frac{3 C_{0}-4 C_{2}+C_{4}}{3 C_{0}-4 C_{1}+C_{2}}\right)-\frac{1}{2} .
$$

In our case, using the number of particles in each slice which appear in Eq. 29, we obtain the values of $C_{i}$ and the estimate $\hat{q}=0.392$. From this value of $\hat{q}$ and the value of $h / T=0.005 / 0.305=0.0164$ we have the constant $\alpha(h / T, q)=34.695$ (see the tables for $\alpha$ in Cruz-Orive, 2006).

Finally, we have all the values to be substituted in Eq. 31 and we obtain $C E^{2}(\hat{N})=0.011$ and $C E(\hat{N})=$ 0.105 , fairly low if we take into account that the gonad is a very irregular domain in the Venerupis pullastra.

In Fig. 4 we have one of the 68 tiles used to estimate oocyte number (see Baddeley and Jensen, 2005, p. 239 and Figure 10.8).

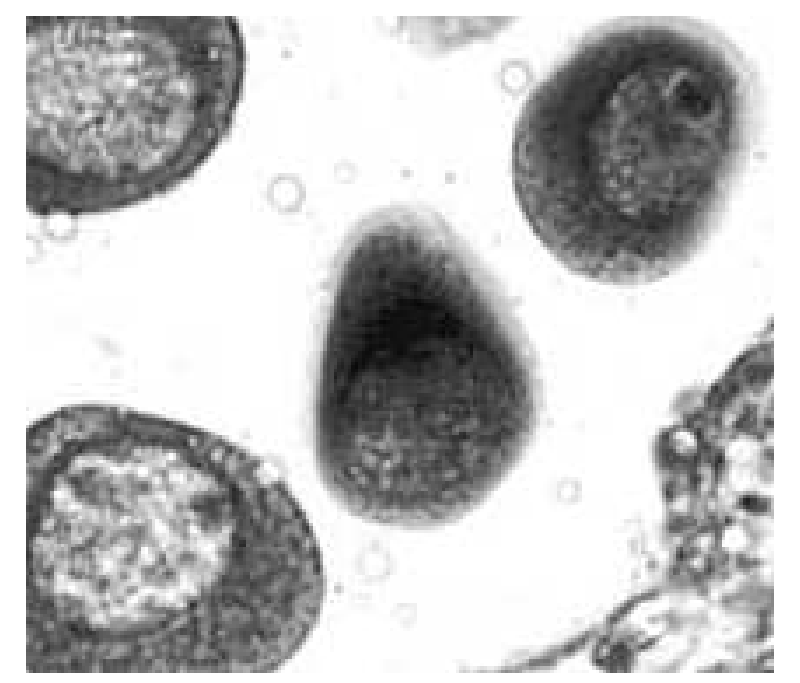

Fig. 4. A tile used to estimate oocyte number with the disector rule. Two oocytes are sampled in this tile.

\section{CONCLUSIONS}

The stereological estimators of gonad volume and oocyte number are effective and easy to implement. Moreover, although the gonad is an irregular structure in the clam, when the section areas of the gonad are estimated by point-counting, there is practically no difference in considering the isotropy condition in the covariogram or not (see Eqs. 14 and 15).

In this paper we have applied stereological methods for estimating the oocyte number in a specimen of Venerupis pullastra. From this study, applying the same methods to several specimens of different native clams, it is expected to obtain conclusions about the fecundity of these types of clams. In addition, our estimation variances will be able to be compared with the biological variance in order to draw inference to biological issues such as assessing fecundity. 


\section{ACKNOWLEDGMENTS}

This work was partially supported by Spanish Ministry of Science and Technology grant MTM200508689-C02-02. The authors are grateful to the reviewers for their comments and suggestions.

\section{REFERENCES}

Baddeley A, Vedel Jensen E B (2005). Stereology for Statisticians. Chapman \& Hall/CRC.

Beukema JJ, Dekker R, Essink K, Michaelis, H (2001). Synchronized reproductive success of the main bivalve species in the Wadden Sea; causes and consequences. Marine Ecology Progress Series 211: 143-55.

Cruz-Orive LM (1993). Systematic sampling is stereology. Bulletin of the International Statistical Institute 55 (2): 451-68.

Cruz-Orive LM (1999). Precision of Cavalieri sections and slices with local errors. J Microsc 193:182-98.
Cruz-Orive LM (2006). A general variance predictor for Cavalieri slices. J Microsc. In press.

Cruz-Orive LM, Geiser M (2004). Estimation of particle number by stereology: an update. J Aerosol Med 17: 197-212.

Cruz-Orive LM, Insausti A, Insausti R, Crespo D (2004). A case study from Neuroscience involving Stereology and Multivariate Analysis. In: . Quantitative Methods in Neuroscience. Ch. 2. Oxford University Press, 16-64.

Gual-Arnau X, Cruz-Orive LM (2006). New variance expressions for systematic sampling: the filtering approach. J Microsc. In press.

Gundersen HJG, Jensen EB (1987). The efficiency of systematic sampling in stereology and its prediction. J Microsc 147:229-63.

Kieu K, Souchet S, Istas J (1999). Precision of systematic sampling and transitive methods. J Statist Plan Inf 77:263-79. 